is then inverted (bacteria side up) and dried on 3MM filter paper (Whatman, Clifton, NJ, USA) until no trace of liquid remains (several seconds). The filter is placed bacteria side down on a Petri dish containing the $\lambda$ TriplEx2 lysis plaques and incubated for $15 \mathrm{~min}$ at $31^{\circ} \mathrm{C}$. A longer incubation time (up to $1 \mathrm{~h}$ ) may be preferable if the lysis plaques are very small. The filter is then removed and placed plaque side up on an LB plate containing at least $100 \mu \mathrm{g} / \mathrm{mL}$ ampicillin and cultured overnight at $31^{\circ} \mathrm{C}$.

Each plaque will give rise to a colony carrying the corresponding plasmid. Single colonies can be replicated in selective medium (Terrific Broth plus antibiotic) or stored as desired. We have used yellow Gilson cones (Gilson, Middletown, WI, USA) to transfer individual colonies into 100 $\mu \mathrm{L}$ of medium in 96-well microtitration plates for culture and storage as glycerol stocks. Two-milliliter cultures were used for plasmid extraction and direct sequencing. More than 12,000 clones have been isolated from three different cDNAs libraries with this method.

To further explore conversion conditions, we have tried other types of support like filter paper or Hybond-N (Amersham Biosciences), but it appears that conversion does not work properly on these supports. On the other hand, it is possible to prepare ready-touse nitrocellulose conversion filters that can be stored. After deposition of bacteria on the filters, we have subjected them to lyophilization and stored them at $4^{\circ} \mathrm{C}$. After 2 days, we have successfully used these filters for conversion without any loss of activity. Thus, it appears that preparation of the conversion filters can be uncoupled from the library conversion itself.

This efficient method of conversion can be applied to other binary vectors, such as $\lambda$ SCREEN $^{\mathrm{TM}}$ and $\lambda$ BlueSTAR $^{\mathrm{TM}}$, both from Novagen (Madison, WI, USA) (3). For the latter vector, which is designed for genomic libraries, the conversion yield cited by Novagen in liquid media is $10 \%$, which is very low. The very high-throughput observed with our technique should greatly increase the utility of the $\lambda$ BlueSTAR vector for production of genomic libraries.

\section{ACKNOWLEDGMENTS}

Funds for this research have been received from the Centre National de la Recherche Scientifique (CNRS) as well as Genoplante.

\section{REFERENCES}

1.Palazzolo, M.J., B.A. Hamilton, D.L. Ding, C.H. Martin, D.A. Mead, R.C. Mierendorf, K.V. Raghavan, E.M. Meyerowitz, et al. 1990. Phage lambda cDNA cloning vectors for substractive hybridization, fusion-protein synthesis and Cre-loxP automatic plasmid subcloning. Gene 88:25-36.

2.Elledge, S.J., J.T. Mulligan, S.W. Ramer, M. Spottswood, and R.W. Davis. 1991. Lambda YES: a multifunctional cDNA expression vector for the isolation of genes by complementation of yeast and Escherichia coli mutations. Proc. Natl. Acad. Sci. USA 88:1731-1735.

3.Doherty, J.P., R. Lindeman, R.J. Trent, M.W. Graham, and D.M. Woodcock. 1993. Escherichia coli host strains SURE and SRB fail to preserve a palindrome cloned in lambda phage: improved alternate host strains. Gene 124:29-35.

4.Williamson, C.L. and R.D. Slocum. 1994. Isolation of cDNA clones by complementation of $E$. coli mutants with infective pBluescript phagemid libraries. BioTechniques 16:986-988.

5.Soderlind, E., A.C. Simonsson, and C.A. Borrebaeck. 1992. Phage display technology in an- tibody engineering: design of phagemid vectors and in vitro maturation systems. Immunol. Rev. 130:109-124.

6.Burg, M.A., K. Jensen-Pergakes, A.M. Gonzalez, P. Ravey, A. Baird, D. Larocca, C.L. Williamson, and R.D. Slocum. 2002. Enhanced phagemid particle gene transfer in camptothecin-treated carcinoma cells. Cancer Res. 62: 977-981.

7.Westwater, C., D.A. Schofield, M.G. Schmidt, J.S. Norris, and J.W. Dolan. 2002. Development of a P1 phagemid system for the delivery of DNA into Gram-negative bacteria. Microbiology 148:943-950

8.Sauer, B.L., inventor. Du Pont de Nemours and Co., assignee. Site-specific recombination of DNA in eukaryotic cells. U.S. patent no. US4,959,317. 1990.

9.Ghosh, K. and G.D. Van Duyne. 2002. CreloxP biochemistry. Methods 28:374-383.

10.Bullock, W.O., J.M. Fernandez, and J.M. Short. 1987. XL1-Blue: a high efficiency plasmid transforming Reca Escherichia coli strain with beta-galactosidase selection. BioTechniques 5:376-379.

Received 16 May 2003; accepted 4 June 2003.

Address correspondence to Jean-Luc Evrard, IBMP-CNRS, 12, rue du Général Zimmer, 67084, Strasbourg Cedex, FRANCE. e-mail: jean-luc.evrard@ibmp-ulp.u-strasbg.fr

\title{
Viability assay for Dictyostelium for use in drug studies
}

\author{
Hannah Alexander, Anthony N. Vomund, and Stephen Alexander \\ University of Missouri, Columbia, MO, USA
}

BioTechniques 35:464-470 (September 2003)

The eukaryotic cells of Dictyostelium discoideum are widely used for studies in cellular and molecular biology (1). The organism has been especially useful for elucidating the molecular mechanisms that underlie cell motility and chemotaxis (2). Recently, D. discoideum has taken on an important new role in investigations of resistance to anticancer drugs and the discovery of new drug targets (3-6). For example, it has been used to identify novel genetic pathways involved in the response to cisplatin (4), and its endogenous morphogen differentiation-inducing factor-1 (DIF-1) has been identified as an antileukemic agent (7). Several features make this organism ideal for these studies, including $(i)$ its well-studied biology and haploid genome, which is almost completely sequenced; (ii) the ability to do homologous gene replacement and random insertional mutagenesis; (iii) the ease and low cost of culture (compared to animal cells) and rapid doubling time; and $(i v)$ its high level of genetic conservation with humans.

Assessing the effects of drugs on living cells requires frequent and accurate measurements of cell viability over several orders of magnitude. D. discoide- 
um is generally grown in axenic liquid medium for molecular biology studies, but it can also feed by the phagocytosis of bacteria on nutrient agar. Viability measurements rely on the latter and are commonly performed by co-plating aliquots of serially diluted cultures with the bacterial food source Klebsiella aerogenes onto $100-\mathrm{mm}$ nutrient agar plates $(8,9)$. The samples are spread on the plate using a glass rod spreader. After incubation for 4 days at $22^{\circ} \mathrm{C}$, the plaques of growing $D$. discoideum appear as clearings in the bacterial lawn. Each plaque represents an initial viable cell and, with appropriate dilutions, one can assess viability over four orders of magnitude. However, this methodology has serious drawbacks that greatly limit quantitation and throughput. These problems center on the expense, difficulty, and accuracy of plating cells on $100-\mathrm{mm}$ agar plates. A typical simple experiment might include four drug concentrations and five 10-fold dilutions of each culture performed in duplicate. This would require 40 plates and $2 \mathrm{~L}$ medium and agar. Spreading 40 plates takes approximately $1 \mathrm{~h}$ and requires experience and finesse on the part of the investigator.

Even with this degree of care, quantitation is limited due to the error introduced by spreading and the difficulty in counting plaques from the more concentrated samples. In general, this requires more dilutions, which requires more plates and more time. Overall, generating viability data over a wide range of drug concentrations is expensive, labor-intensive, and time-consuming, while producing limited data. We have devised a method that obviates all these problems and increases the ability to quantitate viability.

Our method employs 24-well culture plates instead of Petri dishes. Two milliliters of molten SM nutrient agar (9) are dispensed into each well using a repetitive dispensing syringe (Fisher Scientific, Pittsburgh, PA, USA) that fills the wells about half full. The 24well plates are placed on a level surface (a leveled glass plate), and the agar is allowed to harden in the wells. This step ensures that the agar has a level surface with minimal meniscus. It is much faster than pouring plates and uses approximately one-twentieth the amount of medium and agar. Twenty microliters of $K$. aerogenes are added to each well, followed by $15 \mu \mathrm{L}$ serially diluted $D$. discoideum cultures. No spreading is required, which saves time and reduces the error associated with this step. The plates are manually agitated for $1 \mathrm{~min}$ to ensure uniform distribution, left to dry on a level surface, and then incubated at $22^{\circ} \mathrm{C}$. Plating 24 wells takes just a few minutes compared to the 30-40 min needed to plate $24(100 \mathrm{~mm})$ plates and results in more uniform innoculum distribution because it does not require spreading, thus providing better quantitation of clones. Because of the ease and costsavings, series of 2- or 3-fold dilutions can be easily analyzed (compared to standard 10-fold dilutions), which allows for more precise quantitation for drug studies in which changes in sensitivity are often less than one order of magnitude. Moreover, it is feasible to assay more drug concentrations and time points. An unexpected finding is that plaques appear in 2.5-3 days in the 24-well format rather than in 4 days in the $100-\mathrm{mm}$ dishes. Although the underlying reason for this is unclear, it provides timesavings.

Plaques are conveniently viewed by scanning the 24 -well plates with a flat-bed Expression ${ }^{\circledR} 1680$ Scanner (Epson America, Long Beach, CA, USA), which allows the wells to be scored more easily after enlarging and adjusting the contrast of the image (Figure 1). Counting could also be automated using software, although we have not tried that method. Table 1 presents the data from an experiment in which we have measured the viability of a culture of cells in two different dilution series. In each dilution series, three dilutions resulted in easily countable colonies. With only $6 \%$ standard error for the triplicate platings for each dilution series, it is clear that the method provides accurate and reproducible measurements of cell viability.

Figure 2 demonstrates the use of this method to assess the viability of $D$. discoideum cells in response to the chemotherapeutic drug cisplatin. $D$. discoideum cultures $\left(1 \times 10^{6}\right.$ cells $\left./ \mathrm{mL}\right)$ were treated with three different concentrations of cisplatin in two different buffers for $4 \mathrm{~h}$. Fifteen microliters of serial 3-fold dilutions were plated as described earlier, and the plates were scanned after three days. Survival was calculated as the percentage of plaques in the drug-treated cultures relative to the untreated cultures (Figure 2A). These data clearly show that differences in the buffer in which the drug is delivered have a substantial effect on cell viability. Scans of some of the duplicate plates are shown (Figure 2B). It is important to note that viability was measured over four orders of magnitude, precision that is not possible with many viability assays such as dye exclusion or tetrazolium salt [e.g.,3-(4,5dimethylthiazol-2-yl)-2,5-diphenyl tetrasodium, bromide (MTT)]-based assays, which would either require counting 10,000 cells for each data point or is limited by the range of sensitivity of a spectrophotometer.

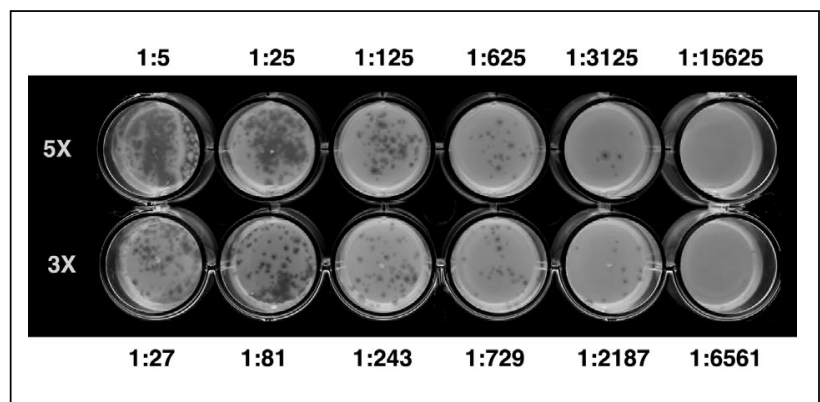

Figure 1. Image of dilution series. Dilution series (3- and 5-fold) in saline solution $\left(10 \mathrm{mM} \mathrm{NaCl}, 10 \mathrm{mM} \mathrm{KCl}, 2 \mathrm{mM} \mathrm{CaCl}{ }_{2}\right.$-distilled water) were made from samples of a culture of logarithmically growing $D$. discoideum cells $\left(1.3 \times 10^{6}\right.$ cells $/ \mathrm{mL}$; assessed by counting cells in a hematocytometer). Fifteen microliters of each dilution were co-plated with $20 \mu \mathrm{L}$ $K$. aerogenes. Standard $K$. aerogenes preparations are made by growing the bacteria in SM medium (9) overnight to $\mathrm{A}_{660}=1.3$, washing in saline, resuspending to one-tenth the original volume, and freezing at $-80^{\circ} \mathrm{C}$ in $15 \%$ glycerol in phosphate-buffered saline (PBS) (Invitrogen, Carlsbad, CA, USA). Aliquots of the bacteria are thawed, pelleted to remove the glycerol, and resuspended in the same volume of SM medium. The plates were incubated at $22^{\circ} \mathrm{C}$, scanned after $68 \mathrm{~h}$ using a flat-bed Expression 1680 scanner, and imaged using Adobe ${ }^{\circledR}$ Photoshop ${ }^{\circledR}$. The images shown were selected from the data presented in Table 1 and are displayed in inverted form, which enhances contrast and enables counting. Dilutions are indicated above and below the corresponding rows of wells. 


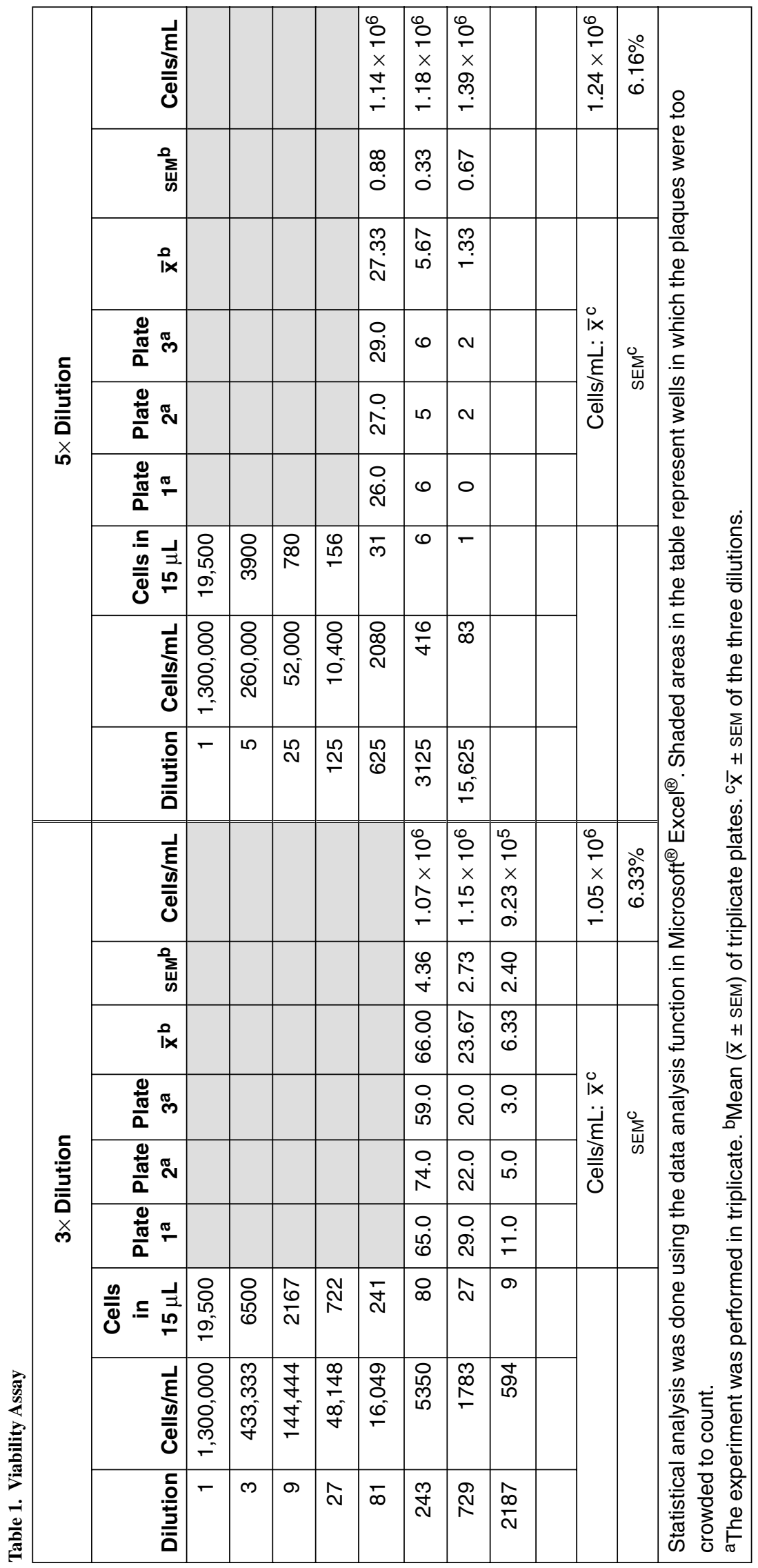


An important aspect of this method is evident when one considers the mechanism of action of many chemotherapeutic drugs that work by damaging the DNA. These drugs often do not kill the cells immediately. Instead, the toxic effect of these drugs is manifest only after one or more cell divisions, when the DNA-drug adducts have interfered with cellular functions such as DNA replication or RNA transcription. To this end, it is necessary to have a method that measures the number of surviving cells that are capable of further cell divisions, as opposed to a method that only detects immediate cell death.

Overall, this method dramatically improves precision and accelerates viability testing with $D$. discoideum. The method could be easily scaled up for highthroughput with the use of robotics to perform the serial dilutions and inoculate the cells into the wells. However, even in its manual form, this method will greatly facilitate the use of this versatile organism in studies of drug resistance, DNA repair, and target discovery.

\section{ACKNOWLEDGMENTS}

This work was supported by the National Institutes of Health grant nos. GM53929 and CA95872 to S.A. and H.A. We thank Cathy Gunther and Junxia Min for their comments on the manuscript and Andy Stegner for his technical help.

\section{REFERENCES}

1.Kessin, R.H. 2001. Dictyostelium-Evolution, Cell Biology, and the Development of Multicellularity. Cambridge University Press, Cambridge, UK.

2.Parent, C.A. and P.N. Devreotes. 1999. A cell's sense of direction. Science 284:765770 .

3.Garcia, M.X.U., C. Roberts, H. Alexander, A.M. Stewart, A. Harwood, S. Alexander, and R.H. Insall. 2002. Methanol and acriflavine resistance in Dictyostelium are caused by loss of catalase. Microbiology 148:333-340.

4.Li, G., H. Alexander, N. Schneider, and S. Alexander. 2000. Molecular basis for resistance to the anticancer drug cisplatin in Dictyostelium. Microbiology 146:2219-2227.

5.Williams, R.S.B., L.L. Cheng, A.W. Mudge, and A.J. Harwood. 2002. A common mechanism of action for three mood-stabilizing drugs. Nature 417:292-295.

6.Yu, S.L., S.K. Lee, H. Alexander, and S. Al-

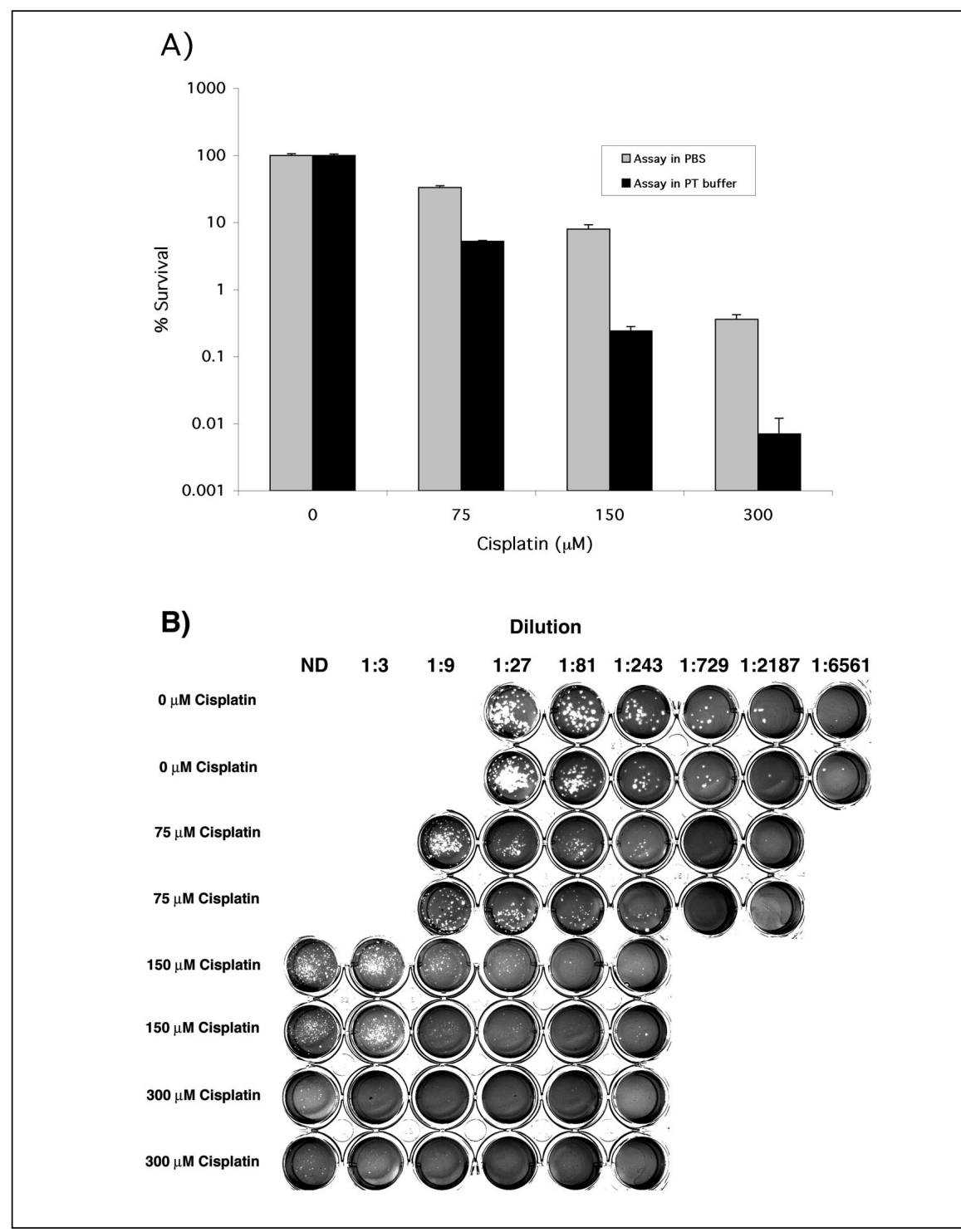

Figure 2. Dose-dependant response of $\boldsymbol{D}$. discoideum to cisplatin. D. discoideum cultures were incubated for $4 \mathrm{~h}$ with the indicated concentration of cisplatin in either phosphate-buffered saline (PBS) or PT buffer ( $3 \mathrm{mM} \mathrm{NaCl}, 1 \mathrm{mM}$ Na-phosphate, $\mathrm{pH}$ 7.4). The cultures were diluted in a 3-fold dilution series, and 15 microliters of each dilution were plated in duplicate in 24-well plates. (A) Bar graph representing the mean percent survival of duplicate dilution series for each cisplatin concentration relative to untreated cultures. Error bars indicate the standard error of the mean (SEM) and are calculated as described in Table 1. (B) Twenty-four well plates from 4-h incubations with cisplatin in PBS. The plates are the data source for the shaded (PBS) bars in panel A. Similar dilutions have been aligned to make viewing easier.

exander. 1998. Rapid changes of nucleotide excision repair gene expression following UV-irradiation and cisplatin treatment of Dictyostelium discoideum. Nucleic Acids Res. 26:3397-3403.

7.Kanai, M., Y. Konda, T. Nakajaima, Y. Izumi, N. Kanda, A. Nanakin, Y. Kubohara, and T. Chiba. 2003. Differentiation-inducing factor-1 (DIF-1) inhibits STAT3 activity involved in gastric cancer cell proliferation via MEK-ERK-dependent pathway. Oncogene 22:548-554.

8.Deering, R. 1988. DNA repair in Dictyostelium. Dev. Genet. 9:483-493.

9.Sussman, M. 1987. Cultivation and synchro- nous morphogenesis of Dictyostelium under controlled experimental conditions. Methods Cell Biol. 28:9-29.

Received 19 May 2003; accepted 4 June 2003.

Address correspondence to Hannah Alexander, Division of Biological Sciences, University of Missouri, Columbia, MO 65211-7400, USA.e-mail: alexanderh@missouri.edu 